\title{
A CLASS OF UNSYMMETRIC STRESS DISTRIBUTIONS IN HELICOIDAL SHELLS*
}

\author{
BY FREDERIC Y. M. WAN (Massachusetts Institute of T'echnology)
}

1. Introduction. In cylindrical coordinates $(r, \theta, z)$, the middle surface of a helicoidal shell is given by the equation $z=a \theta$ where the constant $2 \pi a$ is the pitch of the helicoid. It has been found earlier $[1,2,3,4,5]$ that the solution to a class of elastostatic problems associated with the axial extension and torsion of a thin helicoidal shell requires the displacements of points of the shell to be multivalued in $\theta$ while the strain and stress distributions are independent of $\theta$. The present work is concerned with another class of problems for which the required displacements are multi-valued in $\theta$ while the strain distribution, although single-valued in $\theta$, is no longer axisymmetric. Using a semi-inverse procedure, it is shown in what follows that a certain multi-valued displacement state leads to a univalued but unsymmetric strain state. The class of stress distributions associated with this strain state is then shown to allow the solution of the problem of bending of helicoidal shells by equal and opposite moments applied at the two ends of the shell (Fig. 1).

2. Differential equations. The set of differential equations which governs the elastostatic behavior of the shell is taken from [6] specialized to the case of a helicoidal shell and cylindrical coordinates. The deformation of the shell is completely described in this formulation by three translational displacement components $u, v$, and $w$ in the radial, tangential and normal directions and three rotational displacement components $\phi_{r}, \phi_{\theta}$ and $\omega$ turning about axes in the tangential, radial and normal directions. Four in-plane strain measures $\epsilon_{r}, \epsilon_{r \theta}, \epsilon_{\theta r}$ and $\epsilon_{\theta}$, two transverse shear strains $\gamma_{r}$ and $\gamma_{\theta}$, and four curvature change measures $\kappa_{r}, \kappa_{r \theta}, \kappa_{\theta r}$ and $\kappa_{\theta}$ are defined in terms of the six displacement components by ten strain displacement relations

$$
\begin{array}{ll}
\epsilon_{r}=u^{\prime}, & \epsilon_{r \theta}=v^{\prime}+\frac{w}{R}-\omega, \\
\epsilon_{\theta}=\frac{v^{\prime}}{\alpha}+\frac{r u}{\alpha}, & \epsilon_{\theta r}=\frac{u^{\cdot}}{\alpha}-\frac{r v}{\alpha}+\frac{w}{R}+\omega, \\
\gamma_{r}=\phi_{r}+w^{\prime}-\frac{v}{R}, & \gamma_{\theta}=\phi_{\theta}+\frac{w}{\alpha}-\frac{u}{R}, \\
\kappa_{r}=\phi_{r}^{\prime}+\frac{\omega}{R}, & \kappa_{r \theta}=\phi_{\theta}^{\prime}, \\
\kappa_{\theta}=\frac{r \phi_{r}}{\alpha^{2}}+\frac{\phi_{\theta}}{\alpha}-\frac{\omega}{R}, & \kappa_{\theta r}=\frac{\phi_{r}}{\alpha}-\frac{r \phi_{\theta}}{\alpha^{2}},
\end{array}
$$

*Received April 25, 1966. Preparation of this paper was supported by the Office of Naval Research of the United States Navy. 


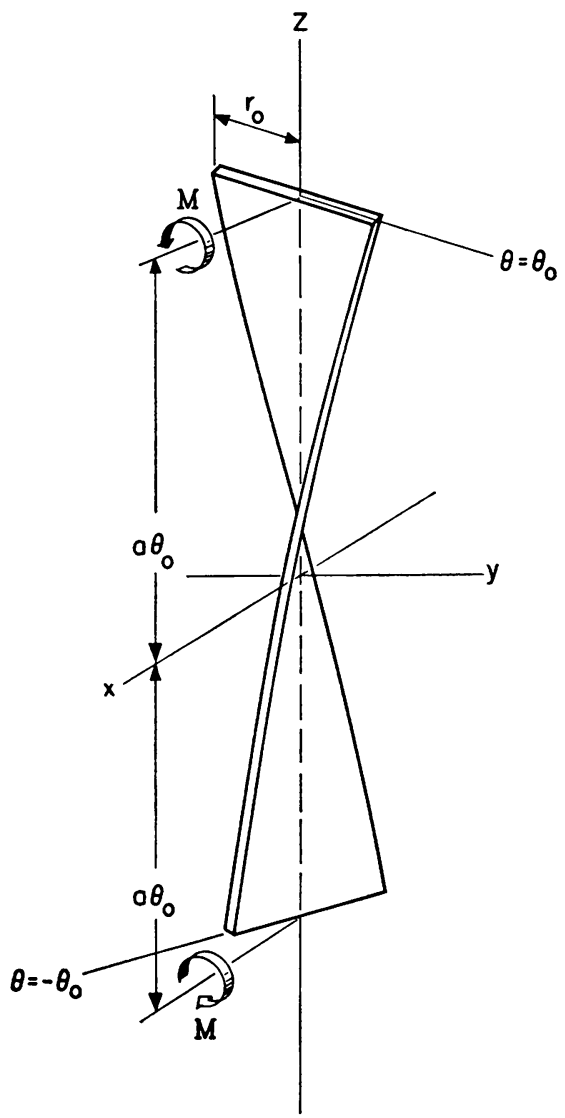

Figure 1.

where primes and dots indicate differentiation with respect to $r$ and $\theta$ respectively, and where

$$
\alpha=\left(a^{2}+r^{2}\right)^{1 / 2}, \quad 1 / R=a / \alpha^{2} .
$$

The ten strain measures are related to the stress measures of the shell by way of a system of stress strain relations. For the sake of definiteness, we assume that these stress strain relations are of the form

$$
\begin{gathered}
N_{r}=\frac{\epsilon_{r}+\nu \epsilon_{\theta}}{A\left(1-\nu^{2}\right)}, \quad N_{\theta}=\frac{\epsilon_{\theta}+\nu \epsilon_{r}}{A\left(1-\nu^{2}\right)}, \\
\epsilon_{r \theta}=\epsilon_{\theta r}=\frac{A(1+\nu)}{2}\left(N_{r \theta}+N_{\theta r}\right), \\
M_{r}=D\left(\kappa_{r}+\nu \kappa_{\theta}\right), \quad M_{\theta}=D\left(\kappa_{\theta}+\nu \kappa_{r}\right), \\
M_{r \theta}=M_{\theta r}=\frac{D(1-\nu)}{2}\left(\kappa_{r \theta}+\kappa_{\theta r}\right), \\
\gamma_{r} \equiv \gamma_{\theta} \equiv 0,
\end{gathered}
$$

where $N_{r}, N_{r \theta}, N_{\theta r}$ and $N_{\theta}$ are the stress resultants and $M_{r}, M_{r \theta}, M_{\theta r}$ and $M_{\theta}$ are 
the stress couples, and where $D$ and $1 / A$ are the bending and stretching stiffness of the shell and $\nu$ is Poisson's ratio.

In the absence of surface loads, the four in-plane stress resultants, $N_{r}, N_{r \theta}, N_{\theta r}, N_{\theta}$, the two transverse shear resultants, $Q_{r}$ and $Q_{\theta}$, and the four stress couples, $M_{r}, M_{r \theta}$, $M_{\theta r}, M_{\theta}$, must satisfy the following equilibrium conditions:

$$
\begin{gathered}
\left(\alpha N_{r}\right)^{\prime}+N_{\theta r}^{\cdot}-\frac{r}{\alpha} N_{\theta}+\frac{a}{\alpha} Q_{\theta}=0, \quad\left(\alpha N_{r \theta}\right)^{\prime}+N_{\theta}^{\cdot}+\frac{r}{\alpha} N_{\theta r}+\frac{a}{\alpha} Q_{r}=0, \\
\left(\alpha Q_{r}\right)^{\prime}+Q_{\theta}-\frac{a}{\alpha}\left(N_{r \theta}+N_{\theta r}\right)=0, \\
\left(\alpha M_{r}\right)^{\prime}+M_{\theta r}-\frac{r}{\alpha} M_{\theta}-\alpha Q_{r}=0, \quad\left(\alpha M_{r \theta}\right)^{\prime}+M_{\theta}+\frac{r}{\alpha} M_{\theta r}-\alpha Q_{\theta}=0, \\
N_{r \theta}-N_{\theta r}+\frac{M_{\theta}-M_{r}}{R}=0 .
\end{gathered}
$$

3. A class of multi-valued displacement functions. Motivated by a solution for the bending of shallow helicoidal shells by end bending moments [7], we consider multivalued displacement functions of the form

$$
\begin{aligned}
u(r, \theta) & =U(r) \sin \theta-\frac{k a^{2}}{4}\left[\left(1+\theta^{2}\right) \sin \theta-\theta \cos \theta\right], \\
v(r, \theta) & =[r V(r)+a W(r)] \frac{\cos \theta}{\alpha}+\frac{k a^{2} r}{4 \alpha}\left(\theta \sin \theta-\theta^{2} \cos \theta\right), \\
w(r, \theta) & =[r W(r)-a V(r)] \frac{\cos \theta}{\alpha}+\frac{k a}{4 \alpha}\left[a^{2} \theta^{2} \cos \theta+\left(a^{2}+2 r^{2}\right) \theta \sin \theta\right], \\
\phi_{r}(r, \theta) & =\Phi_{r}(r) \cos \theta-\frac{k a r}{2 \alpha} \theta \sin \theta, \\
\phi_{\theta}(r, \theta) & =\Phi_{\theta}(r) \sin \theta-\frac{k a}{2}(\theta \cos \theta+\sin \theta), \\
\omega(r, \theta) & =\Omega(r) \cos \theta+\frac{k a^{2}}{2 \alpha} \theta \sin \theta .
\end{aligned}
$$

In these, $U, V, W, \Phi_{r}, \Phi_{\theta}$ and $\Omega$ are functions of $r$ only and $k$ is an arbitrary constant which has the same dimension as the curvature measures. Substituting these expressions into the strain displacement relations, we get

$$
\begin{array}{ll}
\epsilon_{r}=U^{\prime} \sin \theta . & \epsilon_{r \theta}=\left(r V^{\prime}+a W^{\prime}-\alpha \Omega\right) \frac{\cos \theta}{\alpha}, \\
\epsilon_{\theta=}=(r U-r V-a W) \frac{\sin \theta}{\alpha^{2}}, & \epsilon_{\theta r}=(U-V+\alpha \Omega) \frac{\cos \theta}{\alpha}, \\
\gamma_{r}=\left(\alpha \Phi_{r}+r W^{\prime}-a V^{\prime}\right) \frac{\cos \theta}{\alpha}, & \gamma_{\theta}=\left(\alpha^{2} \Phi_{\theta}+a V-a U-r W\right) \frac{\sin \theta}{\alpha^{2}}, \\
\kappa_{r}=\left(\alpha^{2} \Phi_{r}^{\prime}+a \Omega\right) \frac{\cos \theta}{\alpha^{2}}, & \kappa_{r \theta}=\Phi_{\theta}^{\prime} \sin \theta,
\end{array}
$$




$$
\begin{aligned}
\kappa_{\theta r} & =-\left(\alpha \Phi_{r}+r \Phi_{\theta}\right) \frac{\sin \theta}{\alpha^{2}} \\
\kappa_{\theta} & =\left(r \Phi_{r}+\alpha \Phi_{\theta}-a \Omega-k a \alpha\right) \frac{\cos \theta}{\alpha^{2}} .
\end{aligned}
$$

It is apparent that all ten strain measures are single-valued functions of $\theta$.

4. Bending of helicoidal shells by end moments. Consider a helicoidal shell bounded by $r=r_{i}, r=r_{0}$ and $\theta= \pm \theta_{0}$, subject to equal and opposite end bending moments at the axial edges and free of tractions otherwise. For simplicity's sake, we let the axis of the end moments be in the direction of the $x$-axis. The special case with $r_{i}=-r_{0}$ is shown in Fig. 1. Here $-r_{0}$ is taken to mean the image of $r_{0}$ with respect to the $z$-axis.

The stress free condition of the radial edges requires the satisfaction of the KirchhoffBassett conditions

$$
N_{r}+\frac{M_{r \theta}}{R}=N_{r \theta}=M_{r}=Q_{r}+\frac{M_{r \theta}^{\cdot}}{\alpha}=0
$$

at $r=r_{i}$ and $r=r_{0}$.

Force and moment conditions at the axial edges, $\theta= \pm \theta_{0}$, are prescribed in the form

$$
\begin{array}{r}
P_{z}=\int_{r_{i}}^{r_{0}}\left[\frac{a}{\alpha}\left(N_{\theta}+\frac{M_{\theta r}}{R}\right)+\frac{r}{\alpha}\left(Q_{\theta}+M_{\theta r}^{\prime}\right)\right] d r-\left[\frac{r}{\alpha}\left(M_{r \theta}+M_{\theta r}\right)\right]_{r_{i}}^{r_{0}}=0, \\
P_{y}=\int_{r_{i}}^{r_{0}}\left[N_{\theta r} \sin \theta+\frac{r}{\alpha}\left(N_{\theta}+\frac{M_{\theta r}}{R}\right) \cos \theta-\frac{a}{\alpha}\left(Q_{\theta}+M_{\theta r}^{\prime}\right) \cos \theta\right] d r \\
+\left[\frac{a}{\alpha}\left(M_{r \theta}+M_{\theta r}\right) \cos \theta\right]_{r_{i}}^{r_{0}}=0,
\end{array}
$$

$P_{x}=\int_{r_{i}}^{r_{0}}\left[N_{\theta r} \cos \theta-\frac{r}{\alpha}\left(N_{\theta}+\frac{M_{\theta r}}{R}\right) \sin \theta+\frac{a}{\alpha}\left(Q_{\theta}+M_{\theta r}^{\prime}\right) \sin \theta\right] d r$

$$
-\left[\frac{a}{\alpha}\left(M_{r \theta}+M_{\theta r}\right) \sin \theta\right]_{r_{i}}^{r_{0}}=0 \text {, }
$$$$
T_{z}=\int_{r_{i}}^{r_{0}}\left[r N_{\theta}+\frac{r}{R} M_{\theta r}-a Q_{\theta}-a M_{\theta r}^{\prime}\right] \frac{r}{\alpha} d r+\left[\frac{a r}{\alpha}\left(M_{r \theta}+M_{\theta r}\right)\right]_{r_{i}}^{r_{0}}=0 \text {, }
$$$$
T_{\nu}=-\int_{r_{i}}^{r_{0}}\left[M_{\theta} \sin \theta+\left(\frac{r a}{\alpha} N_{\theta}+\frac{r a}{\alpha R} M_{\theta r}+\frac{r^{2}}{\alpha} Q_{\theta}+\frac{r^{2}}{\alpha} M_{\theta r}^{\prime}\right) \cos \theta\right] d r
$$

$$
\begin{array}{r}
+\left[\frac{r^{2}}{\alpha}\left(M_{r \theta}+M_{\theta r}\right) \cos \theta\right]_{r_{i}}^{r_{0}}=0, \\
T_{x}=\int_{r_{i}}^{r_{0}}\left[\left(\frac{r a}{\alpha} N_{\theta}+\frac{r a}{\alpha R} M_{\theta r}+\frac{r^{2}}{\alpha} Q_{\theta}+\frac{r^{2}}{\alpha} M_{\theta r}^{\prime}\right) \sin \theta-M_{\theta} \cos \theta\right] d r \\
-\left[\frac{r^{2}}{\alpha}\left(M_{r \theta}+M_{\theta r}\right) \sin \theta\right]_{r_{i}}^{r_{0}}=M .
\end{array}
$$

The nonintegrated terms in these conditions represent the corner forces introduced by the assumption of vanishing transverse shear strain [8].

The form of stress strain relations (3) and of equilibrium equations (5) shows that 
we can write

$$
\begin{aligned}
& \left(N_{r}, N_{\theta}, M_{r \theta}, M_{\theta r}, Q_{\theta}\right)=\left(n_{r}, n_{\theta}, m_{r \theta}, m_{\theta r}, q_{\theta}\right) \sin \theta \\
& \left(N_{r \theta}, N_{\theta r}, M_{r}, M_{\theta}, Q_{r}\right)=\left(n_{r \theta}, n_{\theta r}, m_{r}, m_{\theta}, q_{r}\right) \cos \theta
\end{aligned}
$$

where $n_{r}, n_{r \theta}, n_{\theta r}, n_{\theta}, q_{r}, q_{\theta}, m_{r}, m_{r \theta}, m_{\theta r}$ and $m_{\theta}$ are functions of $r$ only. The six equilibrium equations can now be written as ordinary differential equations for the $n$ 's, $q$ 's and $m$ 's as follows:

$$
\begin{gathered}
\left(\alpha n_{r}\right)^{\prime}-n_{\theta r}-\frac{r}{\alpha} n_{\theta}+\frac{a}{\alpha} q_{\theta}=0, \quad\left(\alpha n_{r \theta}\right)^{\prime}+n_{\theta}+\frac{r}{\alpha} n_{\theta r}+\frac{a}{\alpha} q_{r}=0 \\
\left(\alpha q_{r}\right)^{\prime}+q_{\theta}-\frac{a}{\alpha}\left(n_{r \theta}+n_{\theta r}\right)=0 \\
\left(\alpha m_{r}\right)^{\prime}+m_{\theta r}-\frac{r}{\alpha} m_{\theta}-\alpha q_{r}=0, \quad\left(\alpha m_{r \theta}\right)^{\prime}-m_{\theta}+\frac{r}{\alpha} m_{\theta r}-\alpha q_{\theta}=0 \\
n_{r \theta}-n_{\theta r}+\frac{m_{\theta}-m_{r}}{R}=0 .
\end{gathered}
$$

Correspondingly, the boundary conditions (8) at the radial edges become

$$
n_{r}+\frac{m_{r \theta}}{R}=n_{r \theta}=m_{r}=q_{r}+\frac{m_{r \theta}}{\alpha}=0
$$

and the integrated conditions (9) at the axial edges become

$$
\begin{aligned}
& \int_{r_{i}}^{r_{0}}\left[\frac{a}{\alpha}\left(n_{\theta}+\frac{m_{\theta r}}{R}\right)+\frac{r}{\alpha}\left(q_{\theta}+m_{\theta r}^{\prime}\right)\right] d r-\left[\frac{r}{\alpha}\left(m_{r \theta}+m_{\theta r}\right)\right]_{r_{i}}^{r_{0}}=0, \\
& \int_{r_{i}}^{r_{0}}\left[n_{\theta r}+\frac{r}{\alpha}\left(n_{\theta}+\frac{m_{\theta r}}{R}\right)-\frac{a}{\alpha}\left(q_{\theta}+m_{\theta r}^{\prime}\right)\right] d r+\left[\frac{a}{\alpha}\left(m_{r \theta}+m_{\theta r}\right)\right]_{r_{i}}^{r_{0}}=0, \\
& \int_{r_{i}}^{r_{0}}\left[n_{\theta r} \cos ^{2} \theta+\left(\frac{a}{\alpha} q_{\theta}+\frac{a}{\alpha} m_{\theta r}^{\prime}-\frac{r}{\alpha} n_{\theta}-\frac{a r}{\alpha} m_{\theta r}\right) \sin ^{2} \theta\right] d r \\
& \left.\left.\int_{r_{i}}^{r_{0}}\left(r n_{\theta}+\frac{r}{R} m_{\theta r}-a q_{\theta}-a m_{\theta r}^{\prime}\right) \frac{r}{\alpha} d r+\left[\frac{a r}{\alpha}\left(m_{r \theta}+m_{\theta r}\right)\right]_{r_{i}}^{r_{0}}=0, m_{\theta r}\right) \sin ^{2} \theta\right]_{r_{i}}^{r_{0}}=0, \\
& \int_{r_{i}}^{r_{0}}\left(\frac{r a}{\alpha} n_{\theta}+\frac{r a}{\alpha R} m_{\theta r}+\frac{r^{2}}{\alpha} q_{\theta}+\frac{r^{2}}{\alpha} m_{\theta r}^{\prime}+m_{\theta}\right) d r-\left[\frac{r^{2}}{\alpha}\left(m_{r \theta}+m_{\theta r}\right)\right]_{r_{i}}^{r_{0}}=0, \\
& \int_{r_{i}}^{r_{0}}\left[m_{\theta} \cos ^{2} \theta-\frac{r}{\alpha}\left(a n_{\theta}+\frac{a}{R} m_{\theta r}+r q_{\theta}+r m_{\theta r}^{\prime}\right) \sin ^{2} \theta\right] d r \\
& \left.+\frac{r^{2}}{\alpha}\left(m_{r \theta}+m_{\theta r}\right) \sin ^{2} \theta\right]_{r_{i}}^{r_{0}}=M .
\end{aligned}
$$

It can now be shown through use of the equilibrium equations (11) and (12) and of the boundary conditions at the radial edges (13) that the five homogeneous integrated conditions in (14) are satisfied identically, while the last condition becomes simply 


$$
M=-\int_{r_{i}}^{r_{0}} m_{\theta} d r
$$

To illustrate the nature of the analysis, we note that through use of the second and third equilibrium equations along with integration by parts, we transform the first condition of (14) to read

$$
\begin{aligned}
\int_{r_{i}}^{r_{0}}\left[\frac{a}{\alpha}\left(n_{\theta}+\frac{m_{\theta r}}{R}\right)+\frac{r}{\alpha}\left(q_{\theta}+m_{\theta r}^{\prime}\right)\right] d r-\left[\frac{r}{\alpha}\left(m_{r \theta}+m_{\theta r}\right)\right]_{r_{i}}^{r_{0}} \\
=\int_{r_{i}}^{r_{0}}\left(\frac{r}{\alpha} q_{\theta}+\frac{a}{\alpha} n_{\theta}\right) d r-\left[\frac{r}{\alpha} m_{r \theta}\right]_{r_{i}}^{r_{0}} \\
=\int_{r_{i}}^{r_{0}}\left[\frac{r}{\alpha} q_{\theta}-\frac{a^{2}}{\alpha^{2}} q_{r}-\frac{r}{\alpha}\left(n_{r \theta}+n_{\theta r}\right)\right] d r-\left[\frac{r}{\alpha} m_{r \theta}+a n_{r \theta}\right]_{r_{i}}^{r_{0}} \\
=\int_{r_{i}}^{r_{0}}\left(-r q_{r}\right)^{\prime} d r-\left[\frac{r}{\alpha} m_{r \theta}\right]_{r_{i}}^{r_{0}}=-\left[r\left(q_{r}+\frac{m_{r \theta}}{\alpha}\right)\right]_{r_{i}}^{r_{0}}
\end{aligned}
$$

where the right hand side vanishes because of the last boundary condition in (13).

5. The two point boundary value problem. The stress strain relations (3) and the three equilibrium equations (12) can be used to express the $n$ 's, $q$ 's and $m$ 's in terms of $U, V$, and $W$. Substituting these expressions into the three force equilibrium equations (11), we have an eighth order system of three ordinary differential equations for the three unknown $U, V$, and $W$. Associated with this eighth order system are four boundary conditions (13) at $r=r_{i}$ and $r=r_{0}$. The solution of this boundary value problem contains the arbitrary constant $k$ which multiplies the multi-valued portion of the displacement state (6). The constant is to be related to the applied moment $M$ by way of the integral condition (15). Carrying out the integration, this becomes a linear relation between $k$ and $M$ which can be written as

$$
M=B k,
$$

where $B$ is a function of $r_{0}, r_{i}, a, \nu, D$ and $A$.

We finally note that our results will, for large values of $a / r_{0}$, tend towards results which have previously been obtained through use of the theory of shallow shells of rectangular plan form [9].

\section{REFERENCES}

1. E. Reissner, On finite bending and twisting of circular ring sector plates and shallow helicoidal shells, Quart. Appl. Math. 11, 473-483 (1953)

2. J. K. Knowles and E. Reissner, Torsion and extension of helicoidal shells, Quart. Appl. Math. 17, 409-422 (1959)

3. E. Reissner, On twisting and stretching of helicoidal shells, Proc. IUTAM, Symposium on Shell Theory, 1959, pp. 434-466, Amsterdam 1960

4. R. G. Sinclair, Axial torsion and extension of helicoidal shells, Ph. D. Thesis, MIT, Sept. 1960

5. F. Y. M. Wan, Twisting and stretching of helicoidal shells, Ph. D. Thesis, MIT, Sept. 1965

6. E. Reissner, Variational considerations for elastic beams and shells, Proc. of ASCE, J. of Eng. Mech., 88, Feb., 1962

7. F. Y. M. Wan, Bending of shallow helicoidal shells, (to appear)

8. A. E. H. Love, $A$ treatise on the mathematical theory of elasticity, 4 th ed, Dover, New York, 1944

9. L. Maunder and E. Reissner, Pure bending of pretwisted rectangular plates, J. Mech. Phys. Solids, 5, 261-266, (1957) 